\title{
Spacetime and vacuum as seen from Moscow
}

\author{
L.B. Okun \\ ITEP, Moscow, 117218, Russia, email: okun@heron.itep.ru
}

\begin{abstract}
An extended text of the talk given at the conference "2001: A Spacetime Odyssey", to be published in the Proceedings of the Inaugural Conference of the Michigan Center for Theoretical Physics, University of Michigan, Ann Arbor, 21-25 May 2001, M.J. Duff and J.T. Liu eds., World Scientific, Singapore, 2002; and of Historical Lecture "Vacuum as seen from Moscow" at the CERN Summer School, 10 August, 2001. Contents: Introduction; Pomeranchuk on vacuum; Landau on parity, P, and combined parity, CP; Search and discovery of $K_{L}^{0} \rightarrow \pi^{+} \pi^{-}$; "Mirror world"; Zeldovich and cosmological term; QCD vacuum condensates; Sakharov and baryonic asymmetry of the universe, BAU; Kirzhnits and phase transitions; Vacuum domain walls; Monopoles, strings, instantons, and sphalerons; False vacuum; Inflation; Brane and Bulk; Acknowledgments; References.
\end{abstract}

\section{Introduction}

This talk presents: a few episodes from the history of ideas on the structure and evolution of vacuum during last 50 years as seen from ITEP; the impact on the theory of vacuum by I.Pomeranchuk, L.Landau, Ya.Zeldovich, A.Sakharov, D. Kirzhnits and their disciples; the importance of international exchange of ideas; the crucial role of experiments on $\mathrm{P}$ and $\mathrm{CP}$ violation; interconnection of particle physics and cosmology.

\section{Pomeranchuk on vacuum}

Being a student of Isaak Pomeranchuk I first heard from him in 1950: "The vacuum is filled with the most profound physical content" and "The Book 
of Physics consists of two volumes: v.1. Pumps and Manometers, v. 2. Quantum Field Theory." In the middle of 1950's Pomeranchuk proved his famous theorem about equality of particle and antiparticle cross-sections at the same target. It is not an accident that the Regge pole with quantum numbers of vacuum, which is responsible for this equality, was called later Pomeranchuk pole or pomeron.

In 1956 Pomeranchuk encouraged Boris Ioffe, Alexei Rudik and myself to work on P-non-conservation. By applying CPT theorem [1] to the famous paper of T.D. Lee and C.N. Yang [2] we came to the conclusions [3] that: 1) $\vec{\sigma} \vec{p}$ correlations suggested by them would signal not only $\mathrm{P}-$, but also Cviolation. 2) $K_{S}$ and $K_{L}$ are respectively even and odd under $\mathrm{T}$ (or, better, $\mathrm{CP}$ ), but not under C [4]. Hence $K_{S} \not \rightarrow 3 \pi^{0}, K_{L}^{0} \rightarrow 3 \pi^{0}$ (November 1956). The same conclusions were reached by T.D. Lee, R. Oehme and C.N. Yang [5] (January 1957) (see Nobel lectures by T.D. Lee and C.N. Yang [6, 7] (December 1957)).

\section{Landau on parity, $P$, and combined parity, CP}

Till the end of 1956 Lev Landau did not believe in the possibility of Pviolation. In the summer of 1956 he did not encourage Iosif Shapiro to publish a Moscow University report [8], in which an experiment of the type carried out later by C.S.Wu et al. was suggested (according to Shapiro's report energy of a system changed its value under mirror reflection). Landau was convinced that $\mathrm{P}$ must be conserved because vacuum is mirror symmetric. (One would not expect this argument from an author of 1950 article with Ginzburg [9] in which spontaneous symmetry breaking was introduced into theory of superconductivity.)

We (IOR) discussed our paper with Landau. A few weeks after it was submitted to JETP, Landau [10] put forward (December 1956) the idea of absolute CP-invariance and conservation of "combined parity". According to this idea the "sin" of P-violation was committed by particles and antiparticles (with opposite signs), but the mirror symmetry of vacuum was preserved.

The idea was widely accepted. I considered it to be beautiful, but on the other hand, I did not understand why the Lagrangian could not have complex coefficients. Therefore in lectures given at ITEP (1960-61) and Dubna (1961) 
[11] and in the book [12 based on these lectures (1963) the importance of experimental tests of $\mathrm{CP}$ was stressed, in particular search for $K_{L} \rightarrow 2 \pi$.

\section{Search and discovery of $K_{L}^{0} \rightarrow \pi^{+} \pi^{-}$}

A special search at Dubna was carried out by E. Okonov and his group. They have not found a single $K_{L}^{0} \rightarrow \pi^{+} \pi^{-}$event among 600 decays of $K_{L}^{0}$ into charged particles [13] (Anikina et al., JETP, 1962). At that stage the search was terminated by administration of the Lab. The group was unlucky.

Approximately at the level 1/350 the effect was discovered by J.Christensen, J.Cronin, V.Fitch and R.Turlay [14 at Brookhaven in 1964 in an experiment the main goal of which was $K_{L} \rightarrow K_{S}$ regeneration in matter.

Thus absolute CP-invariance was falsified.

\section{5 "Mirror world"}

Still the appeal of Landau's idea of absolutely symmetric vacuum was so strong that in 1965 Igor Kobzarev, Isaak Pomeranchuk and myself suggested the hypothesis of a "mirror world" [15]. We assumed CPA invariance, where $A$ [from "Alice through the Looking Glass"] transforms our part of the Lagrangian into its mirror part.

Each of our particles has its mirror counterpart. The mirror particles have between them the same electromagnetic, weak and strong interactions as ours. In principle there might exist mirror nuclei, atoms, molecules, stars, planets, galaxies, even mirror life. Whether they actually exist depends on cosmological evolution.

The possibility of the existence of both "left protons", $p_{L}$, and "right protons", $p_{R}$, had been discussed by T.D. Lee and C.N. Yang in two last paragraphs of their famous article [2]. But they believed that $p_{L}$ and $p_{R}$ can interact with the same pion and the same photon. We have proved that this is impossible.

According to our original assumption, the only particles which belong to both our and mirror worlds are gravitons. If there were two gravitons, nothing would connect the two worlds and the idea of a mirror world would have no physical consequences.

Why the graviton but not, say, a photon? As soon as you assume that the 
photon is common to both worlds, you immediately come to contradiction with experiments. Colliding $e^{+} e^{-}$through a virtual photon would annihilate not only into our particles but also into mirror ones. Besides, the electromagnetic radiative corrections would be doubled destroying the beautiful agreement of QED with experiment.

In 1982 I studied the oscillations between our photons and sterile ones, "paraphotons" [16]; in 1983 - considered a stronger coupling between two worlds which could be caused by exchange of some hypothetical neutral bosons [17]. A number of authors considered mirror particles as a substantial component of dark matter. Many enthusiastic papers about mirror matter were written recently by R. Foot (see [18] and references therein) without proper citation of ref. [15].

\section{Zeldovich and cosmological term}

Yakov Zeldovich was very excited when he realized that a non-vanishing $\Lambda$ term is strongly required by quantum field theory. In 1967 he estimated $\varepsilon_{\Lambda}$ on the basis of pairs of virtual elementary particles in vacuum. First he started with $\varepsilon_{\Lambda} \sim m_{P l}^{4}$ and found enormous (124 orders) discrepancy with observations (private communication). Then 19 he assumed that quartically divergent loops $\left(\sim m_{P l}^{4}\right.$ and even $\left.m^{4}\right)$ vanish and estimated

$$
\varepsilon_{\Lambda} \sim \frac{m^{6}}{m_{P l}^{2}},
$$

where $m$ is the mass of a typical elementary particle of the order of a proton mass $(1 \mathrm{GeV})$. That was the first attempt to put $m_{P l}^{2}$ in denominator. (The contribution of the loop is proportional to gravitational interaction.) In order to bridge the gap of $10^{8}$, he tried

$$
\varepsilon_{\Lambda} \sim G G_{F} m^{8}
$$

where $G$ is Newton constant $\left(m_{P l}=G^{-1 / 2}\right)$, while $G_{F}$ is Fermi constant.

In 1968 Zeldovich noticed the negative sign of the contribution of fermions and indicated that the contributions of bosons and fermions might cancel each

other! 20] This was before Yuri Golfand and his student Evgeni Likhtman published [21] the first paper on supersymmetry.

Recently Johannes Blümlein and Paul Söding kindly informed me about references [22, 23], in which early unpublished estimates made by young W. 
Pauli are described. Pauli discussed vacuum fluctuations and their influence on cosmology. Note that he overlooked that cosmological term would lead to inflation and concluded that the universe would be smaller than the distance from the earth to the moon. Even earlier, in 1916, the "zero point energy" had been considered by W. Nernst ([24], see also [25]).

\section{$7 \quad$ QCD vacuum condensates}

Here I will make a digression from cosmology to Quantum Chromodynamics (QCD) with its quarks and gluons.

In $1965 \mathrm{~V}$. Vanyashin and M. Terentyev [26] discovered the negative sign of the vacuum polarization of gauge bosons in the case of $\mathrm{SU}(2)$ gauge symmetry. Their numerical result was slightly incorrect, as the "ghost" was unknown at that time. A correct number (with the same negative sign!) was derived by I. Khriplovich in 1969 by using Coulomb gauge [27]. The negative sign of vacuum polarization was a novelty: for photons in QED it is positive. But at that time the nonabelian theories with gauge vector bosons were not considered seriously at ITEP. Even after SLAC experiments on deep inelastic scattering and their parton interpretation by J. Bjorken [28] and R. Feynman [29] we were not clever enough to realize the importance of negative vacuum polarization.

The situation has drastically changed in 1973 when H. Fritzsch, M. GellMann, and H. Leutwyler [30 suggested QCD with its SU(3) color gauge symmetry. In papers by H.D. Politzer 31 and D.J. Gross and F. Wilczek [32] the negative sign was rediscovered, and its importance for QCD was stressed: it lead to decreasing color charge at short distances (and hence to asymptotic freedom at high momentum transfers).

It should be noted that Vanyashin and Terentyev [26] considered as "absurd" not the ultraviolet, but the infrared behaviour of the charge where its square first rises and then becomes negative. At present we know that this region is never reached: in Electroweak Model because of the Higgs mechanism, in QCD because of confinement. The increase of charge at large distances makes perturbation theory invalid at such distances. Non-perturbative effects manifest themselves in non-vanishing vacuum expectation values (VEVs) of colorless products of quark and gluon fields, the so-called QCD condensates. These condensates were used at ITEP by Michael Shifman, Arkady Vainshtein and Valentin Zakharov in order to successfully describe the properties 
of various hadrons [33]. This work was followed by many hundreds of theoretical papers (for a review see [34]).

\section{Sakharov and baryonic asymmetry of the universe, BAU}

In 1967 I had the privilege to witness the creation of Sakharov's seminal paper [35] on the Baryonic Asymmetry of the Universe (BAU). He started out from the CP-violating charge asymmetry of the semileptonic decays of $K_{L}^{0}$ and from the nonequality of branching ratios in the decays of $\Sigma^{+}$and $\widetilde{\Sigma^{+}}$discussed by S.Okubo [36]. Using it as a springboard, he jumped from strange particles to the universe and from strangeness violation to baryon number violation. A small excess of baryons over antibaryons in the hot big bang soup survived, while the bulk annihilated into photons, neutrinos and antineutrinos. Photons are observed today as CMBR. The cosmic background neutrinos are tantalizing. When devising the baryon nonconserving ad hoc interaction Sakharov was helped by ITEP team. In the framework of SM and its extensions there are several mechanisms of baryon number violation; among them grand unification [37] and triangle anomalies [38]. The latter mechanism raises serious problems [39] at electroweak scale: it might wipe out baryon asymmetry, unless the original lepton asymmetry is involved [40]. The problem of BAU can not be solved in the framework of Standard Model. (V. Rubakov and M. Shaposhnikov [41].)

\section{$9 \quad$ Kirzhnits and phase transitions}

With the advent of the Standard Model based on $S U(3) \times S U(2) \times U(1)$ gauge symmetry new concepts came to particle physics; one of them is the vacuum expectation value $(\mathrm{VEV})$ of the Higgs field. It reduces $S U(2)_{L} \times U(1)_{Y}$ to $U(1)_{e m}$ and provides with masses leptons, quarks, $W$ and $Z$ bosons. An important step was made 42] in 1972 by David Kirzhnits, a theorist at the Lebedev Institute, who realized that the Higgs VEV is a function of temperature and has to vanish in the early universe, when we go backwards in time to temperatures much higher than the present value of VEV. Hence the masses of particles have also to vanish. This idea was further developed by Kirzhnits and his student Andrei Linde [43] and later by many others 


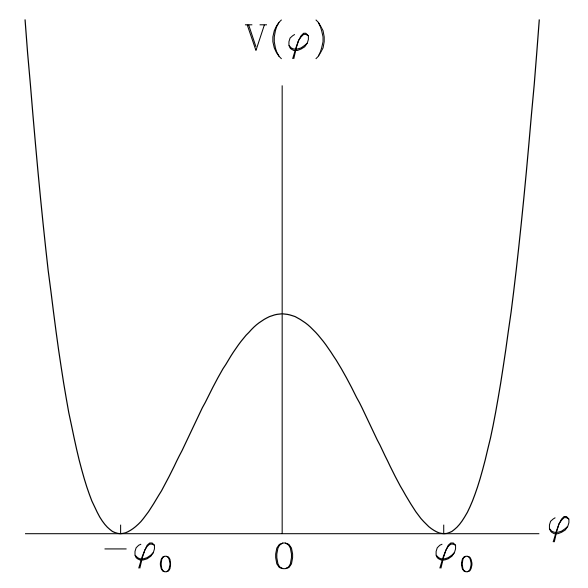

Figure 1:

[44]. (Both A. Sakharov and D. Kirzhnits were disciples of Igor Tamm.) I first heard about the vacuum phase transition from David on a street during a conference in Tashkent in 1972.

\section{Vacuum domain walls}

Two years later Igor Kobzarev, Yakov Zeldovich and myself [45] merged the idea of vacuum phase transition with a model of spontaneous $\mathrm{CP}$ violation proposed by T.D.Lee [46]. According to this model a neutral pseudoscalar field has two degenerate vacua (see Fig. 1).

The vacuum around us has a sign that was fixed by chance during the cooling of the universe and formation of VEVs. But at any distant enough place the other sign might have been chosen. The domains of different sign are separated from each other by "domain walls" the thickness and density of which are determined by VEVs of the field $\varphi$ and by its self-coupling. Typical electroweak scale is several hundred GeV. We discussed the evolution of the universe filled with domain walls and concluded that at present the nearest wall should have gone beyond the horizon, leaving as a farewell an anisotropy of CMBR. The advent of inflational cosmology (see below) greatly weakened 
this argument against spontaneous CP-violation.

\section{Monopoles, strings, instantons, and sphalerons}

Vacuum domain walls were the first megascopic cosmological elementary objects considered in the framework of quantum field theory. They were caused by spontaneous breaking of discrete symmetry. Later in 1974 G.'t Hooft 47] and A.Polyakov [48] discovered magnetic monopoles as solutions of spontaneously broken SU(2) gauge symmetry. Cosmological creation of monopoles and of strings (the latter appear as solutions of a broken U(1) gauge symmetry) was considered in 1976 by T.W.B.Kibble 49. (Microscopic U(1) strings of Nambu type underlying dual resonance model of hadrons were considered in 1973, without any reference to cosmology, by H.B. Nielsen and P. Olesen [50], who were inspired by Abrikosov's vertices in a superconductor [51].)

Since the early 1980's a whole "industry of theoretical papers" on cosmological role of domain walls, strings and monopoles appeared. I will mention here only articles by Ya.B. Zeldovich [52], A. Vilenkin [53] and an exotic example of a cosmic string invented by Albert Schwartz [54 in 1982 after he learned from me about the "mirror world". A particle, after making a circle around his "Alice string", transforms into a mirror particle and becomes invisible for a terrestrial observer [55].

A special type of non-perturbative transitions between topologically different vacua of non-abelian gauge fields were constructed by A. Belavin, A. Polyakov, A. Schwartz, V. Tyupkin [56] in 1975 and by 'Hooft [57 in 1976. The latter called them instantons. In the four-dimensional eucledian space (with imaginary time) instantons represent solutions with finite action. Many attempts to understand the mechanism of confinement in QCD have exploited instantons.

Amplitudes of high temperature transitions between vacua in electroweak theory, called sphalerons, were first analyzed by N. Manton 58 in 1983 and by F. Klinkhamer and N. Manton [59] in 1984 in connection with baryon nonconserving phase transition at electroweak scale. 


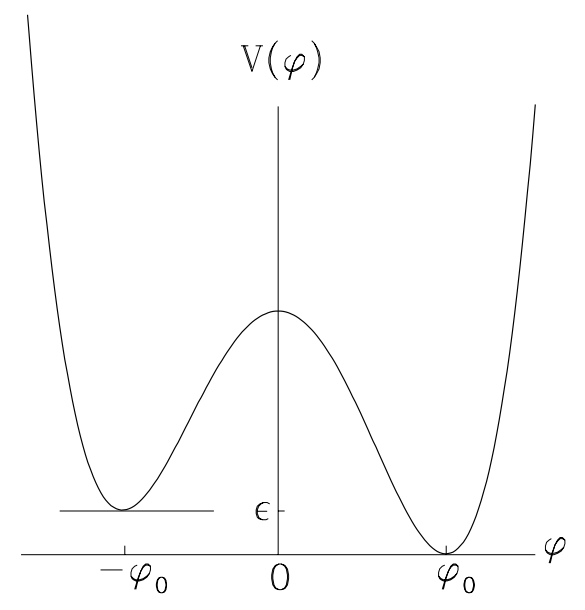

Figure 2:

\section{False vacuum}

In 1974 T.D.Lee and G.C.Wick [60] suggested a Higgs potential with two vacua, one of which lies a little bit higher than the other (see Fig. 2).

The cosmological consequences for universe that lives in an upper metastable vacuum were elaborated [61] by Igor Kobzarev, Mikhail Voloshin and myself in 1974 and later, in 1977, by Curt Callan and Sidney Coleman [62, 63] (they dubbed it "false vacuum") and also by Paul Frampton 64].

The spontaneous decay of a false vacuum starts by formation through quantum tunneling of the smallest bubble of the true vacuum surrounded by a wall which separates the two vacua. The critical radius of this bubble is such that the gain of energy proportional to its volume becomes large enough to compensate the mass of the wall which is proportional to its surface:

$$
\frac{4 \pi}{3} R_{c}^{3} \cdot \varepsilon=4 \pi R_{c}^{2} \sigma
$$

where $\varepsilon$ is the energy density of the false vacuum (see Fig. 2), while $\sigma$ is the surface density of the wall. After that the bubble expands classically, destroying the universe.

When I first thought that the creation of a bubble could be catalyzed at a collider, my back shivered. Then I reassured myself: all possible collisions 
have already occurred in the early universe. A few months later I told Andrei Sakharov about the bubble. His reaction was: "Such theoretical work should be forbidden". My argument about collisions in the early universe was rejected by him: "Nobody had collided two nuclei of lead".

In 1984 P.Hut 65 published an estimate of uranium-uranium collisions in cosmic rays during the time of the existence of universe. It turned out that the number of such collisions, $N$, was many orders of magnitude larger than at any future heavy ion collider: $N \sim 10^{47}$ for Fe with $E \geq 100 \mathrm{GeV} /$ nucleon. Experiments at RHIC (BNL) study $\mathrm{Au}-\mathrm{Au}$ collisions at $\sqrt{s} \sim 200 \mathrm{~A} \mathrm{GeV}$ $\sim 40 \mathrm{TeV}$ ( $A \simeq 197$ for gold). There will be $2 \cdot 10^{11}$ collisions in 10 years, first at $30 \mathrm{GeV}$, then at $70 \mathrm{GeV}$, and finally at $100 \mathrm{GeV} /$ nucleon. A fixed target NA 50 experiment at CERN gathered $2 \cdot 10^{12} \mathrm{~Pb}-\mathrm{Pb}$ collisions at $\sqrt{s} \sim 17 \mathrm{~A}$ $\mathrm{GeV} \sim 3.5 \mathrm{TeV}(A \simeq 207$ for lead). The experiment ALICE at LHC (CERN) will study $\mathrm{Pb}-\mathrm{Pb}$ collisions at $\sqrt{s} \sim 1200 \mathrm{TeV}$. The abundances of heavy nuclei may be $10^{-5}-10^{-10}$ of that of $\mathrm{Fe}$, which still leaves a huge safety factor in cosmic rays. For a recent update of these arguments see 66, 67].

\section{Inflation}

The notion of a false vacuum with large energy density (VEV) naturally led Alan Guth 68 to the idea of inflationary universe (1980). The exponential expansion solves the problems of flatness and the horizon as well as homogeneity and isotropy problems. It also explains the vanishing abundance of magnetic monopoles. (For earlier models of exponential expansion of the universe see papers by E.B. Gliner [69] and A.A. Starobinsky [70].)

In 1982 Andrei Linde [71] and independently Andreas Albrecht and Paul Steinhardt [72 suggested how to stop this expansion by producing many vacuum bubbles and high entropy. Also in 1982 Alexander Dolgov [73 suggested a mechanism with a rolling scalar field which led to what later was dubbed "quintessence".

Recent measurements with high angular resolution of CMBR fluctuations confirm the inflation scenario (experiments BOOMERANG [74], MAXIMA [75], DAST [76]). The flatness of the universe is corroborated by observations of high-z supernova of type Ia [77, 78]. On the other hand, they give a puzzling result: the fractional energy density of the universe $\Omega=\varepsilon / \varepsilon_{c}$, where $\varepsilon_{c}$ is the critical density, corresponding to the flat universe, is dominated by cosmological term - i.e. energy density of vacuum $(\sim 70 \%)$, then follows the 
so-called dark matter $(\sim 30 \%)$, while ordinary baryonic matter constitutes only about $3 \%$. To understand the cause and mechanism of such fine tuning is a great challenge.

During the 1990s the development of inflation cosmology has lead to a new view on the place of our universe in the world. According to this view, our universe is a part of eternal metauniverse, which consists of innumerable universes. They create their offsprings by forming Big-Bang bubbles in false vacua. The properties of vacua, fields, particles and even number of spacetime dimensions differ from one universe to the other 81]. This enormous variety may explain the anthropic properties of our universe which is so nicely tuned for our existence (see, e.g. [82]).

\section{Brane and bulk}

The idea of extra dimensions [83, 84] has been actively discussed for 60 years before it was first combined with the idea of "domain walls" in these extra dimensions [79], [80]. According to this idea, our four-dimensional world is a "brane" in a multidimensional bulk 86] - 88]. All known particles and their fields are confined (completely, or almost completely) within the brane, while real and virtual gravitons are allowed to leave the brane and to propagate in the bulk. This drastically changes gravity. The Planck scale may become as low as $1 \mathrm{TeV}$. There could be deviations from the standard Newton's force at distances of the order of millimeters and many other strange phenomena. But at present all this is a kind of a daydreaming.

\section{Acknowledgments}

I am grateful for their hospitality to Mike Duff who invited me to the Inaugural Conference of the MCTP and to Guido Altarelli and Marcella Diemoz who invited me to give the Historical Lecture at the CERN Summer School. 


\section{References}

[1] W. Pauli, in Niels Bohr and the Development of Physics, edited by W. Pauli, London, Pergamon Press, 1955.

[2] T.D. Lee and C.N. Yang, Phys. Rev. 104 (1956) 254.

[3] B.L. Ioffe, L.B. Okun and A.P. Rudik, Zh.ETF 32 (1957) 396 [ Sov. Phys. JETP 5 (1957) 328].

[4] M. Gell-Mann and A. Pais, Phys. Rev. 97 (1955) 1387.

[5] T.D. Lee, R. Oehme and C.N. Yang, Phys. Rev. 106 (1957) 340.

[6] C.N. Yang, in Nobel lectures. Physics 1942 - 1962 (Elsevier, 1964), p. 393.

[7] T.D. Lee, in Nobel lectures. Physics 1942 - 1962(Elsevier, 1964), p. 406.

[8] I.S. Shapiro, Moscow State University report, Summer 1956, unpublished.

[9] V.L. Ginzburg and L.D. Landau, ZhETF 20 (1950) 1064 (in Russian).

[10] L.D. Landau, ZhETF 32 (1957) 405 [Sov. Phys. JETP 5 (1957) 336]; Nucl. Phys. 3 (1957) 127.

[11] L.B. Okun, Lectures on theory of weak interactions of elementary particles, 16 ITEP preprints, 1960 - 1961 (in Russian).

English translations: Theory of weak interactions: Thirteen lectures, AEC-tr-5226. US Atomic Energy Commision. Oak Ridge, Tenn. Another English translation of 13 lectures: NP-10254; of lectures 14-16: NP-10254, 10842, 10845, 10840.

Revised edition: Preprint JINR P-833, Dubna, 1961 (in Russian).

[12] L.B. Okun, Weak Interactions of Elementary Particles (Pergamon, 1965) (a translation of Slaboe vzaimodeystvie elementarnych chastits (Fizmatgiz, Moskva, 1963); another translation: Israel Program Sci. Translations, 1965.

[13] M. Anikina et al., ZhETF 42 (1962) 130, [Sov. Phys. JETP 15 (1962) 93]. 
[14] J.H. Christenson, J.W. Cronin, V.C. Fitch and R. Turlay, Phys. Rev. Lett. 13 (1964) 138.

[15] I.Yu. Kobzarev, L.B. Okun and I.Ya. Pomeranchuk, Yad. Fiz. 3 (1966) 1154 [Sov. J. Nucl. Phys. 3 (1966) 837].

[16] L.B. Okun, ZhETF 83 (1982) 892; JETP 56 (1982) 502.

[17] L.B. Okun, On Search for mirror particles, Preprint ITEP-149 (1983).

[18] R. Foot, Seven (and a half) reasons to believe in mirror matter, astro$\mathrm{ph} / 0102294$.

[19] Ya.B. Zeldovich, Pisma ZhETF 6 (1967) 883.

[20] Ya.B. Zeldovich, Uspekhi Fiz. Nauk 95 (1968) 209.

[21] Yu.A. Golfand, E.P.Likhtman, Pis'ma ZhETF 13 (1971) 323.

[22] C.P. Enz, A. Thellung, Helv. Phys. Acta 33 (1960) 839.

[23] N. Straumann, The mystery of the cosmic vacuum energy density and the accelerated expansion of the Universe, astro-ph/9908342.

[24] W. Nernst, Verhandlungen der deut. physik. Gesellschaft 13 (1916) 83.

[25] S.E. Rugh, H. Zinkernagel, The quantum vacuum and the cosmological constant problem, hep-th/0012253.

[26] V.S. Vanyashin, M.V. Terentyev, ZhETF 48 (1965) 565; JETP 21 (1965) 375.

[27] I.B. Khriplovich, Yad. Fiz. 10 (1969) 409; Sov. J. Nucl. Phys. 10 (1970) 235.

[28] J.D. Bjorken, A theorist view on $e^{+} e^{-}$-annihilation, in: Proc. Intern. Symposium on Electron and Photon Interactions at High Energy, August 1973. Preprint SLAC-PUB-1318.

[29] R.P. Feynman, Photon-Hadron interactions, W.A. Benjamin Inc. 1972.

[30] H. Fritzsch, M. Gell-Mann, H. Leutwyler, Phys. Lett. 47B (1973) 365. 
[31] H.D. Politzer, Phys. Rev. Lett. 30 (1973) 1346.

[32] D. Gross and F. Wilczek, Phys. Rev. Lett. 30 (1973) 1343.

[33] M. Shifman, A. Vainshtein and V. Zakharov, Nucl. Phys. B147 (1979) $385 ; 448$.

[34] M.A. Shifman, ITEP Lectures on Particle Physics and Field Theory, World Scientific Publishing Co. (1999).

[35] A.D.Sakharov, ZhETF Pis'ma 5 (1967) 32, [JETP Lett. 5 (1967) 24].

[36] S. Okubo, Phys. Rev. 109 (1958) 984.

[37] H. Georgi, Grand unified theories, in: History of Original Ideas and Basic Discoveries in Particle Physics, Ed. Harvey B. Newman and Thomas Ypsilantis, Plenium Press, New York and London, 1996.

[38] G. 't Hooft, Phys. Rev. Lett. 37 (1976) 8.

[39] V.A. Kuzmin, V.A. Rubakov and M.E. Shaposhnikov, Phys. Lett. B155 (1985) 36.

[40] M. Fukugita and T. Yanagida, Phys. Lett. B174 (1986) 45.

[41] V. Rubakov and M. Shaposhnikov, UFN 166 (1996) 493; Phys. Usp. 39 (1996) 461.

[42] D.A.Kirzhnitz, Pisma Zh.ETF 15 (1972) 745, [JETP Lett. 15 (1972) 529].

[43] D.A. Kirzhnitz and A.D. Linde, Phys. Lett. 42B (1972) 471.

[44] A.D. Linde, Particle Physics and Inflationary Cosmology (1990), Fizika Elementarnykh Chastits i Inflatsionnaya Kosmologiya, (Nauka, Moscow, 1990).

[45] I.Yu. Kobzarev, L.B. Okun and Ya.B. Zeldovich, Zh.ETF 67 (1974) 3 [JETP 40 (1975) 1]; Phys. Lett. B50 (1974) 340.

[46] T.D. Lee, Phys. Reports 9C (1974) No.2.

[47] G. 't Hooft, Nucl. Phys. B79 (1974) 276. 
[48] A.M. Polyakov, ZhETF Pis'ma 20 (1974) 276, [JETP Lett. 20 (1974) 194].

[49] T.W.B. Kibble, J. Phys. A9 (1976) 1387.

[50] H.B. Nielsen, P. Olesen, Nucl. Phys. B61 (1973) 45.

[51] A.A. Abrikosov, ZhETF 32 (1957) 1442.

[52] Ya. B. Zeldovich, Mon. Not. Roy. Astron. Soc. 192 (1980) 663.

[53] A.Vilenkin, Phys. Lett. 46 (1981) 1169.

[54] A.S. Schwarz, Nucl. Phys. B208 (1982) 141.

[55] A.S. Schwarz and Yu.S. Tyupkin, Nucl. Phys. 209 (1982) 427.

[56] A.A. Belavin, A.M. Polyakov, A.S. Schwartz and Yu.S. Tyupkin, Phys. Lett. 59B (1975) 85.

[57] G. 't Hooft, Phys. Rev. Lett. 37 (1976) 8; 3432; Phys. Rev. D14 (1976) 3432.

[58] N.S. Manton, Phys. Rev. D28 (1983) 2019.

[59] F.R. Klinkhamer, N.S. Manton, Phys. Rev. D30 (1984) 2212.

[60] T.D. Lee and G.C. Wick, Phys. Rev. D9 (1974) 2291.

[61] M.B. Voloshin, I.Yu. Kobzarev and L.B. Okun, Yad. Fiz. 20 (1974) 1229; [Sov. J. Nucl. Phys. 20 (1975) 644].

[62] S. Coleman, Phys. Rev. D15 (1977) 2929; Err. D16 (1977) 1248.

[63] C. Callan, S. Coleman, Phys. Rev. D16 (1977) 1762.

[64] P.H. Frampton, Phys. Rev. D15 (1977) 2922.

[65] P. Hut, Nucl. Phys. A418 (1984) 301C.

[66] R.L. Jaffe, W. Busza, J. Sandweiss, and F. Wilczek, Review of Speculative "Disaster Scenarios" at RHIC, hep-ph/9910333, 2000.

[67] A. Dar, A. De Rujula and U. Heinz, Phys. Lett. B470 (1999) 142. 
[68] A.H. Guth, Phys. Rev. D23 (1981) 347.

[69] E.B. Gliner, ZhETF 49 (1965) 542.

[70] A.A. Starobinssky, Pisma ZhETF 30 (1979) 719; Phys. Lett. B91 (1980) 99.

[71] A.D. Linde, Phys. Lett. 108B (1982) 389.

[72] A. Albrecht, P.J. Steinhardt, Phys. Rev. Lett. 48 (1982) 1220.

[73] A.D. Dolgov, An attempt to get rid of the cosmological constant, in: Proc. Very early Universe, Nuffield Workshop, Cambridge, 1982, 449458. Cambridge Univ. Press, 1983.

[74] C.B. Netterfield et al., astro-ph/0104460.

[75] A.T. Lee et al., astro-ph/0104459.

[76] N.W. Halverson, astro-ph/0104489.

[77] B. Schmidt et al., Astrophys. J. 507 (1998) 46.

[78] S. Perlmutter et al., Astrophys. J. 517 (1999) 563.

[79] V.A. Rubakov and M.E. Shaposhnikov, Phys. Lett. B125 (1983) 136.

[80] K. Akama, in Gauge Theory and Gravitation, Proceedings of the International Symposium, Nara, Japan, 1982, eds. K. Kikkawa, N. Nakanishi and H. Nariai (Springer-Verlag, 1983).

[81] A. Linde, Lectures in inflationary cosmology, hep-th/9410082.

[82] L.B. Okun, Uspekhi Fiz. Nauk 161 (1991) 177, Sov. Phys. Uspekhi 34 (1991) 818.

[83] T. Kaluza, Sitzungsberichte Preussische Akad. Wissenschaften (1921) 966.

[84] O. Klein, Z. Phys. 37 (1926) 895.

[85] P. Horava and E. Witten, Nucl. Phys. B460 (1996) 506.

[86] G. Dvali and M. Shifman, Phys. Lett. B396 (1997) 64. 
[87] L. Randall and R. Sundrum, Phys. Rev. Lett. 83 (1999) 4690.

[88] N. Arkani-Hamed, S. Dimopoulos, and G. Dvali, Phys. Lett. B429 (1998) 263. 